\title{
Comparison Between Robust PID and Predictive PI Controllers with Constrained Control Signal Noise Sensitivity
}

\author{
P. Larsson, T. Hägglund \\ Department of Automatic Control, Lund University, Lund, Sweden. \\ e-mail: perola|tore@control.lth.se
}

\begin{abstract}
A performance comparison between PID and predictive PI (PPI) controllers, i.e., two different prediction methods, is presented. Optimization of controller and measurement filter parameters, considering load disturbance rejection, robustness and noise sensitivity, is performed for a batch of industrially representative processes. For a majority of the processes and the constraints chosen, results show that the performances of the controllers are similar. However, the PID controller yields better performance for processes where increased phase and gain may be achieved over a wider frequency interval than what is possible by the PPI controller.
\end{abstract}

\section{INTRODUCTION}

Low-level controllers in the process industry are required to have few tuning parameters, be easy to tune manually, and the parameters should affect the control loop in an intuitive manner. The most common control structure in process industry is the PID structure, and most often is the derivative action turned off, resulting in a PI controller. This is due to the introduction of noise sensitivity by the derivative part, but also because it requires tuning and additional filtering. Adding derivative action may increase performance significantly and joint design of PID controller and measurement filter for load rejection, where limitations on noise sensitivity may be set, have recently emerged, see for instance Kristiansson and Lennartson [2006], Garpinger [2009], Larsson and Hägglund [2011].

Adding derivative action to a PI controller yields phase advance, i.e., prediction capability. Another type of prediction is given by a Smith predictor, see Smith [1957]. The Smith predictor structure contains a model of the process without dead-time, which is used for simulation internally with the control signal as input. If the model is accurate, its output is a prediction of the process output with the prediction horizon equal to the process deadtime. However, adding a predictor structure increases the number of controller parameters significantly and adds to the operational complexity. A simplified form of the Smith predictor structure with a $\mathrm{PI}$ controller, denoted $\mathrm{PI}_{\tau}$ or predictive PI (PPI), has the same number of parameters as the PID controller and can be tuned manually in an analogous manner, see Shinskey [1994] and Hägglund [1996].

Performance comparisons between the PID and the PI controller with a Smith predictor structure, regarding load disturbances, have been made in e.g., Kristiansson and Lennartson [2001] and Ingimundarson and Hägglund [2002]. In this paper, comparison between the performance of the PID and the PPI controller will be considered. The differences to the before mentioned references are that the compared control structures have the same number of tuning parameters and the comparison is performed in a discrete time setting. Additionally, both robustness towards process uncertainty and control signal noise sensitivity are considered using other types of measures.

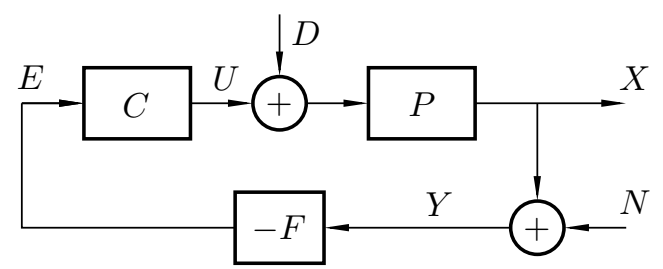

Fig. 1. Closed loop control structure with process $P$, controller $C$ and measurement filter $F$.

\section{FEEDBACK AND CONTROLLER STRUCTURES}

The closed loop considered in the comparison is shown in Figure 1, where $P, C$ and $F$ are the process, controller and measurement filter, respectively. The sensor signal $Y$, giving information about the process output $X$, is corrupted by measurement noise $N$. The load disturbance $D$ is assumed to enter on the process input together with the control signal $U$, which is calculated by the controller from the control error $E$.

The considered PID controller is on parallel form with the input-output relation

$$
U=K\left(1+\frac{1}{s T_{i}}+s T_{d}\right) E
$$

where $K, T_{i}$ and $T_{d}$ are proportional gain, integral time and derivative time, respectively. The comparison will be made in a discrete time setting and the integral and derivative parts are discretized using forward and backward differences, respectively, with sampling period $h$.

The input-output relation of the PPI controller is

$$
\begin{aligned}
U & =K\left(1+\frac{1}{s T_{i}}\right)\left(E-\frac{K^{-1}}{s T_{i}+1}\left(1-e^{-s L}\right) U\right) \\
& =K\left(1+\frac{1}{s T_{i}}\right) E-\frac{1}{s T_{i}}\left(1-e^{-s L}\right) U,
\end{aligned}
$$

where $K, T_{i}$ and $L$ are the proportional gain, integral time and controller dead-time, respectively. Compared to a PI controller with Smith predictor, the process model in the PPI controller is parametrized with gain $K^{-1}$, time constant $T_{i}$ and dead-time $L$. Thus, only for certain values of the parameters is the PPI controller equal to a PI controller with a Smith predictor using a first order model with dead-time, i.e., model matching. The PPI controller is not limited to model matching and its performance can in 


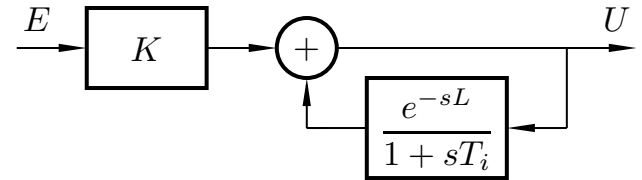

Fig. 2. Implementation of the predictive PI controller.

general be improved if model matching is not considered, see Shinskey [2001]. Due to the model parametrization, the PPI controller handles integrating processes, no additional filters are required as for the Smith predictor structure.

The input-output relation of the PPI controller shows that an ordinary PI controller acts on the control error $E$ and the prediction, due to the process model parametrization, is performed by low-pass filtering the control signal $U$. The transfer function of the PPI controller can be factorized as $C_{0} C_{\text {pred }}$, where $C_{0}$ is a PI controller and $C_{\text {pred }}$ is a predictor structure as

$$
C_{0}=K\left(1+\frac{1}{s T_{i}}\right), \quad C_{\text {pred }}=\frac{1}{1+\frac{1}{s T_{i}}\left(1-e^{-s L}\right)} .
$$

The predictor behavior is essentially determined by the ratio $L / T_{i}$ and its gain tends to 1 at high frequencies. Thus, the PPI controller gain tends to $K$.

The PPI controller may be implemented by introducing the dead-time $L$ in the positive feedback in a PI controller implementation, see Figure 2. Discretization is made by zero-order hold of the positive feedback transfer function.

To achieve desired noise sensitivity level of the feedback, measurement filters should be designed together with the controllers, see Isaksson and Graebe [2002]. In Larsson and Hägglund [2011], it was shown that a second order filter with damping $1 / \sqrt{2}$ is preferable to a first order filter for a PID. To achieve the same roll-off in the feedback when using a PPI controller, a first order filter will be used together with the PPI. The filters are parametrized by the time constant $T_{f}$ as

$$
F_{\mathrm{PPI}}=\frac{1}{s T_{f}+1}, \quad F_{\mathrm{PID}}=\frac{1}{s^{2} T_{f}^{2}+\sqrt{2} T_{f} s+1},
$$

and are discretized using zero-order hold. Thus, both the design of a PID controller and a PPI controller, with their associated measurement filters, will have four parameters to determine.

\section{COMPARISON OF PREDICTION METHODS}

The two controllers presented in the previous section perform prediction in two different ways. A Taylor series expansion of the time-domain control error $e\left(t+T_{d}\right)$ is

$$
e\left(t+T_{d}\right) \approx e(t)+T_{d} \frac{d e(t)}{d t}+\ldots
$$

The two first terms is the linear prediction performed jointly by the proportional and derivative part of the PID controller. The prediction in the PPI controller is performed by $C_{\text {pred }}$, which has the following Taylor series expansion for small $s$, see Åström and Hägglund [2006],

$$
C_{\text {pred }}=\frac{1}{1+L / T_{i}}\left(1+\frac{1}{2} \frac{\left(L / T_{i}\right)^{2}}{1+L / T_{i}} T_{i} s+\ldots\right) .
$$

In Figure 3, the transfer function $C_{\text {pred }}$, with $T_{i}=0.25$ and $L=1$, is shown together with a PD controller with $K$ and $T_{d}$ set using the Taylor series expansion of $C_{\text {pred }}$ as
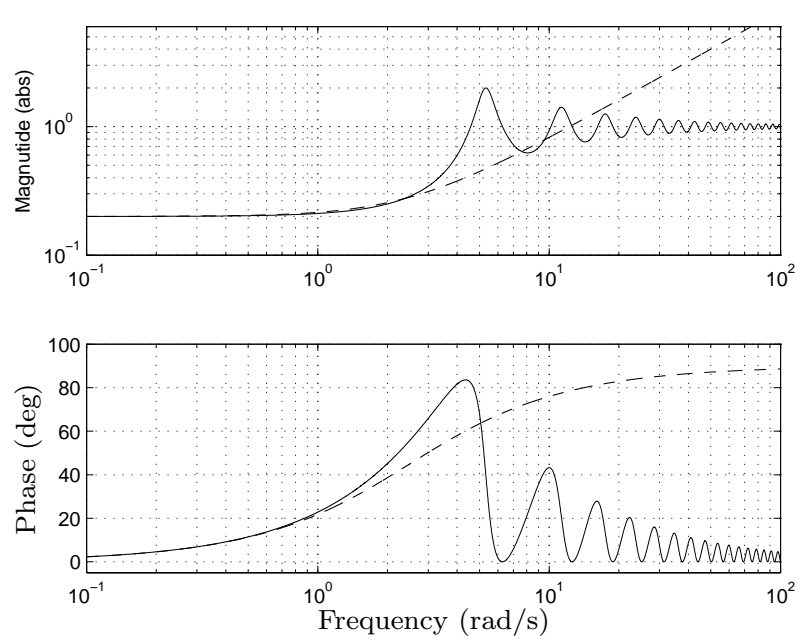

Fig. 3. The predictor $C_{\text {pred }}$ in the PPI controller (-) and a PD controller (--).

$$
K=\frac{1}{1+L / T_{i}}, \quad T_{d}=\frac{1}{2} \frac{\left(L / T_{i}\right)^{2}}{1+L / T_{i}} T_{i}
$$

The predictor $C_{\text {pred }}$ has a distinct phase advance peak, associated with a peak in the gain. The phase advance falls rapidly after the peak. The PD controller has an increasing phase over a wider frequency interval, associated with a steadily increasing gain. As noted in the previous section, the gain of $C_{\text {pred }}$ tends to 1 at high frequencies, while the gain of the PD controller tends to infinity, hence the orders of $F_{\mathrm{PPI}}$ and $F_{\mathrm{PID}}$. Adding the measurement filters to the predictors, the phase advances will be smaller and gains lower, but the differences in characteristics remain.

\section{ROBUSTNESS AND NOISE SENSITIVITY}

Robustness towards process uncertainties is imperative to consider at controller design. The Smith predictor structure, and thus also the PPI controller, is sensitive towards modeling errors in the process dead-time, see Palmor [1980]. A method to reduce the sensitivity, presented in Normey-Rico et al. [1997], is to add a low-pass filter in the feedback structure. This approach requires at least one parameter in the added filter to be set, yielding undesirable increase in design complexity. Another method was presented in Kristiansson and Lennartson [2001], where an upper gain limit on the open-loop transfer function is set after the phase cross-over frequency. In Ingimundarson and Hägglund [2002], the open-loop gain was instead limited to be strictly less than 1 after the gain cross-over frequency. The last two methods may be conservative as they imply that any reduction in process dead-time will never yield instability, even though bounds on the deadtime uncertainty may be known.

The robustness measures for process uncertainties to be used in this paper were presented in Larsson and Hägglund [2009] for continuous time systems and can be used for discrete time systems with negligible approximation errors if an appropriate sample period is used, see Appendix A. The measures consider upper amplitude limits on $S_{\Delta}$ and $T_{\Delta}$, which are the closed loop sensitivity and complementary sensitivity functions extended to depend on the process dead-time uncertainty $\Delta L$. Constraints may be set as 


$$
\begin{aligned}
\left\|S_{\Delta}(\Delta L)\right\|_{\infty} & \leq M_{S} \\
\left\|T_{\Delta}(\Delta L)\right\|_{\infty} & \leq M_{T},
\end{aligned}
$$

which should hold for all considered $\Delta L$ in an interval $\Delta \underline{L} \leq \Delta L \leq \Delta \bar{L}$, where $\Delta \underline{L}$ and $\Delta \bar{L}$ and lower and upper bounds on the dead-time uncertainty. Thus, the constraints guarantee that the maximum gains of the sensitivity functions $S$ and $T$ are less than or equal to $M_{S}$ and $M_{T}$, respectively, when the process dead-time is changed within the interval. The parameters $M_{S}$ and $M_{T}$ may be specified to set desired robustness towards process gain and time constant uncertainties. See Larsson and Hägglund [2009] for a method to calculate the largest interval, which includes 0 , for $\Delta L$ in Eqs. (1)-(2) when a nominal Nyquist curve and $M_{S}$ and $M_{T}$ are given.

Measurement noise may yield undesirable activity of the control signal. Assuming the noise is white with zero mean and with variance, i.e., energy, $\sigma_{n}^{2}$, then the constraint

$$
\|C F S\|_{2} \leq \eta_{u}
$$

where $S$ is the sensitivity function, limits the control signal energy due to measurement noise to $\sigma_{u}^{2} \leq \eta_{u}^{2} \sigma_{n}^{2}$. Rapid variations in the control signal are also undesirable from an actuator point of view. In Larsson and Hägglund [2011], a measure was presented that considers the inter-sample amplitude of the control signal, i.e., derivative, due to measurement noise. It may be expressed as

$$
\left\|\Delta_{z} C F S\right\|_{2} \leq \eta_{\Delta u}
$$

where $\Delta_{z}$ is the difference operator, i.e., $\Delta_{z}=(z-1) / z$. Both the above constraints on noise sensitivity of the control signal will be used in the design of controllers and measurement filters.

\section{OPTIMIZATION FORMULATION}

The controllers and measurement filters will be compared using the integrated absolute error (IAE) at a load disturbance step as performance measure. The design optimization problem, with the constraints in the previous section, may be stated as follows,

$$
\begin{aligned}
\underset{\mathcal{K}}{\operatorname{minimize}} & h \sum_{k=0}^{\infty}|x(k)| \\
\text { subject to } & \left\|S_{\Delta}(\Delta L)\right\|_{\infty} \leq M_{S}, \quad \Delta \underline{L} \leq \Delta L \leq \Delta \bar{L} \\
& \left\|T_{\Delta}(\Delta L)\right\|_{\infty} \leq M_{T}, \quad \Delta \underline{L} \leq \Delta L \leq \Delta \bar{L} \\
& \|C F S\|_{2} \leq \eta_{u} \\
& \left\|\Delta_{z} C F S\right\|_{2} \leq \eta_{\Delta u} \\
& \underline{T}_{f} \leq T_{f} \leq \bar{T}_{f},
\end{aligned}
$$

where $\mathcal{K}$ contains controller and measurement filter parameters, $x(k)$ is the process output when a load disturbance step is applied at initial time, and $h$ is the sample period. The constraints on the extended sensitivity functions are required to hold for all dead-time uncertainties $\Delta L$ that are in the interval defined by $\Delta \underline{L}$ and $\Delta \bar{L}$. The lower limit on $T_{f}$ is set to $\underline{T}_{f}=h / \pi$ due to sampling. The upper limit $\bar{T}_{f}$ is set such that the filter break point is at a higher frequency than the frequency where derivative action or prediction by $C_{\text {pred }}$ is begun. That is, the filter may not be used for loop-shaping at low- and mid-frequencies, only for attenuation at high frequencies, i.e., as a measurement filter. For the PPI controller, the inverse of the lowest frequency where the controller amplitude curve has a positive derivative, denoted $\omega_{f}$, is taken as an upper limit and may be calculated numerically in the optimization. For PID control, the limit is taken as the inverse of the largest modulus of the controller zeros of the continuous time transfer function. Thus, for $F_{\mathrm{PPI}}$ and $F_{\mathrm{PID}}$, respectively, the upper limits are

$$
\bar{T}_{f}=\frac{1}{\omega_{f}}, \text { and } \bar{T}_{f}=\left|\frac{1}{2 T_{d}}+\sqrt{\frac{1}{4 T_{d}^{2}}-\frac{1}{T_{i} T_{d}}}\right|^{-1} .
$$

The optimization problems are solved in MATLAB ${ }^{\circledR}$ using the Control System Toolbox ${ }^{\text {TM }}$, Optimization Toolbox ${ }^{\text {TM }}$, and Simulink ${ }^{\circledR}$.

\section{PROCESS BATCH AND DESIGN PARAMETERS}

Controllers and filters have been optimized for the following batch of 82 industrially representative processes,

$$
\begin{aligned}
P_{1} & =\frac{e^{-s}}{s T+1}, T=0.1,0.2,0.3,0.5,1,1.5,4,10,50 \\
P_{2} & =\frac{e^{-s}}{(s T+1)^{2}}, T=0.1,0.2,0.3,0.5,1,2,6,10,50 \\
P_{3} & =\frac{1}{(s+1)(s T+1)^{2}}, T=0.05,0.1,0.2,0.5,2,5,10 \\
P_{4} & =\frac{1}{(s+1)^{n}}, \quad n=3,4,5,6,7,8 \\
P_{5} & =\frac{1}{\Pi_{k=0}^{3}\left(\alpha^{k} s+1\right)}, \quad \alpha=0.4,0.5, \ldots, 0.9 \\
P_{6} & =\frac{e^{-s L_{1}}}{s\left(s T_{1}+1\right)}, \quad P_{7}=\frac{e^{-s L_{1}}}{(s T+1)\left(s T_{1}+1\right)}, \quad T=1,10 \\
L_{1} & =0.02,0.05,0.1,0.3,0.5,0.7,0.9,1, L_{1}+T_{1}=1 \\
P_{8} & =\frac{1-\alpha s}{(s+1)^{3}}, \quad \alpha=0.1,0.2, \ldots, 1.1 \\
P_{9} & =\frac{1}{(s+1)\left((s T)^{2}+1.4 s T+1\right)}, \quad T=0.1,0.2, \ldots, 1 .
\end{aligned}
$$

The batch includes both lag-dominated, delay-dominated, oscillative and integrating processes as well as processes with inverse step responses and are all sampled using zeroorder hold. The sample period used both for processes and control structures is $h=0.02$, i.e., small enough for the differences in used discretization methods not to effect the result. In all designs, $M_{S}=M_{T}=1.4$, which is a common value when considering upper limits on the sensitivity functions, see Åström and Hägglund [2006]. The noise sensitivity constraints are set to $\eta_{u}=\eta_{\Delta u}=1$, see Larsson and Hägglund [2011] for design choices. For simplicity, if the considered process has a dead-time $L$, then $\Delta \underline{L}=-L / 10$ and $\Delta \bar{L}=L / 10$. If the process is modeled without dead-time, only an additive dead-time uncertainty is considered by $\Delta \underline{L}=0$ and $\Delta \bar{L}=\tilde{L} / 10$, where $\tilde{L}$ is the apparent dead-time of the process.

\section{BATCH RESULTS}

Ratios between the resulting IAE for the PPI and PID structures are found in Figure 4 as functions of the normalized process dead-time $\tau$. For a majority of the processes, the performances of the two prediction methods are similar, yielding ratios within 0.9-1.1. Comments on the specific processes are given below.

For the PPI structure and $P_{1}$ and $P_{2}$ with $\tau>0.5$, it is only the constraint on $\left\|S_{\Delta}\right\|_{\infty}$ that is active, limiting the peak gains. For the PID structure, the corresponding 
limit is $\tau>0.8$. For these processes, a derivative part may give gain increase and phase advance over a wider frequency interval than the PPI predictor, which yields a better performance for the PID structure. See Example I in Section 8, where the designs for $P_{1}$ with $T=0.3$ are shown in detail.

At $\tau$ close to $0, P_{1}$ is essentially a first-order process, requiring no phase advance in the feedback for high lowfrequency gain and thus low IAE. Resulting feedbacks are basically PI controllers with measurement filters and the constraints on $\|C F S\|_{2}$ and $\left\|S_{\Delta}\right\|_{\infty}$ are active.

For higher order processes, phase advance in the feedback may increase performance significantly. For $P_{2}$ and $P_{3}$ with large time constants, i.e., $T \geq 10$, and thus small $\tau$, phase advance should be made at low frequencies for high performance. As the constraint functions $\|C F S\|_{2}$ and $\left\|\Delta_{z} C F S\right\|_{2}$ are calculated over all frequencies and essentially only over high frequencies, respectively, significant gain increase in the open-loop can be given in desired frequency interval as the measurement filter can decrease the gain sufficiently at high frequencies. This benefits the PID structure, which can have phase advance and gain increase over a wider frequency interval than the PPI structure, and has therefore significantly better performance for these processes. Both control structures have the constraints on $\left\|S_{\Delta}\right\|_{\infty}$ and $\|C F S\|_{2}$ active. However, the PID controller has higher gain than the PPI controller at low frequencies, and the PPI controller has higher gain than the PID controller at high frequencies.

In comparison with $P_{2}$ and $P_{3}$ with $T \geq 10$, the other non-integrating processes with $0<\tau<0.5$, except for $P_{1}$, have essentially smaller time constants. Thus, the phase advance should be at higher frequencies. Here, the phase advance and gain of the derivative part may not be used over a significantly wider frequency interval than the interval with phase advance and gain given by the PPI predictor, resulting in similar performances of the two controller structures. In general, both structures have the constraint on $\left\|S_{\Delta}\right\|_{\infty}$ active for the considered processes. The PID structure has always $\|C F S\|_{2}$ on the constraint, but never $\left\|\Delta_{z} C F S\right\|_{2}$. The PPI structure has in general a smaller $T_{f}$ than the PID structure, resulting in a higher high-frequency gain and either $\|C F S\|_{2}$ or $\left\|\Delta_{z} C F S\right\|_{2}$ active. This will be seen in Example II below, where $P_{9}$ with $T=0.3$ is considered.

The open-loop transfer function for the integrating processes $P_{6}$ has an initial phase of $-\pi \mathrm{rad} / \mathrm{s}$, requiring phase advance to rotate the Nyquist curve away from the robustness constraints. This can be made over a wider frequency interval by the PID structure than by the PPI structure, resulting in a significantly better performance for the PID structure. Both control structures have $\left\|S_{\Delta}\right\|_{\infty}=\left\|T_{\Delta}\right\|_{\infty}=1.4$, while the PID structure has $\|C F S\|_{2}$ active for all values of $L$ and the PPI structure has $\left\|\Delta_{z} C F S\right\|_{2}$ active only for $L<0.3$. Thus, the PPI structure cannot give enough phase advance for $L \geq 0.3$ over a wide frequency interval such that the gain can be increased and yield the control signal constraints active.

\section{DESIGN EXAMPLES}

Table 1 shows the resulting IAE, parameter and constraint function values for Example I and II below. Nyquist dia-

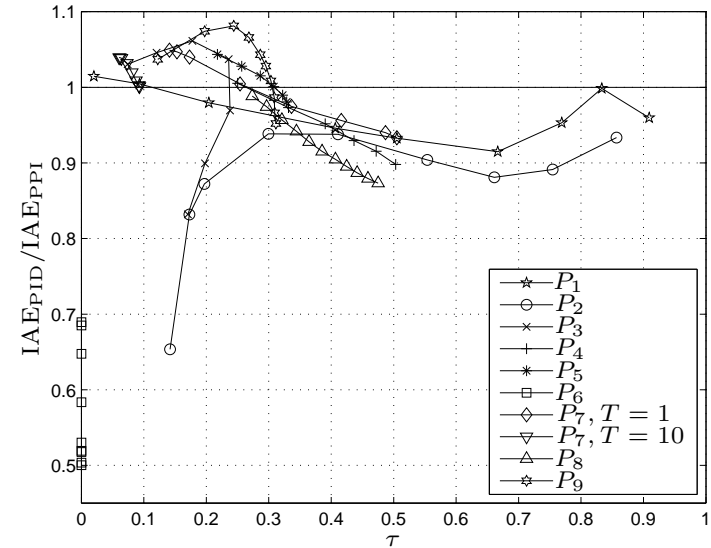

Fig. 4. Ratios between IAE for PID and PPI feedback structures as a function of normalized dead-time $\tau$ for the processes in the batch.

grams, Bode diagrams of the feedbacks and load disturbance step responses are shown in figures 5-7 for Example I and in figures 8-10 for Example II. In the load disturbance response simulations, the measurement signal is in the second half corrupted with zero mean white noise with a standard deviation of 0.025 .

\subsection{Example $I: P_{1}, T=0.3$}

The optimal PPI controller with measurement filter for $P_{1}$ with $T=0.3$ was not able to give sufficient gain for the noise sensitivity constraints to be active, yielding the filter time constant to be at its lower bound. The presented result for the PPI controller is therefore from an optimization without measurement filter.

To yield phase advance, the PPI controller makes gain peaks, resulting in loops in the Nyquist diagram. Prior the phase cross-over frequency, $\Delta L=\Delta \bar{L}$ yields $\left\|S_{\Delta}\right\|_{\infty}=$ $M_{S}$, while the two first gain peaks yield $\left\|S_{\Delta}\right\|_{\infty}=M_{S}$ for $\Delta L=\Delta \underline{L}$. Hence, the robustness constraint limits the loop gain to be even larger, and hence also the phase advance of the predictor. After the two first peaks, the open loop tends to 0 as the gain of the predictor tends to 1 . The PPI controller is not able to increase its gain at higher frequencies, and the noise sensitivity constraints are not active. The optimal controller parameters are not equal to model-matched design.

The PID controller follows the initial phase advance of the PPI controller, but the gain does not have, nor is able to have, a distinct peak at mid frequencies. Prior the phase cross-over frequency, $\Delta L=\Delta \bar{L}$ yields $\left\|S_{\Delta}\right\|_{\infty}=M_{S}$. After the phase cross-over frequency, the PID controller can increase its gain, yielding phase advance over a wide frequency interval. As the process gain decreases with frequency, the PID gain increases, making the Nyquist curve to be almost circular and $\left\|S_{\Delta}\right\|_{\infty}=M_{S}$ at the negative real axis. At higher frequencies, the measurement filter yields roll-off such that the noise sensitivity constraints are met. The control signal from the PID controller, compared to the PPI controller, reacts faster when the load disturbance is seen in the measurement signal and is also more sensitive to measurement noise.

The maximum phase advance and associated gain are higher for the PPI structure than for the PID structure. However, for this example, phase advance and gain in- 
Table 1. IAE, feedback parameter and constraint function values in Example I and II.

\begin{tabular}{cccccccccccc}
\hline Process & Controller & IAE & $K$ & $T_{i}$ & $T_{d}$ & $L$ & $T_{f}$ & $\left\|S_{\Delta}\right\|_{\infty}$ & $\left\|T_{\Delta}\right\|_{\infty}$ & $\|C F S\|_{2}$ & $\left\|\Delta_{z} C F S\right\|_{2}$ \\
\hline \multirow{2}{*}{$P_{1}, T=0.3$} & PPI & 2.17 & 0.60 & 0.17 & - & 1.1 & - & 1.40 & 1.00 & 0.61 & 0.84 \\
& PID & 2.10 & 0.29 & 0.61 & 0.30 & - & 0.034 & 1.40 & 1.00 & 1.0 & 0.89 \\
\hline \multirow{2}{*}{$P_{9}, T=0.3$} & PPI & 0.825 & 2.0 & 0.46 & - & 0.98 & 0.042 & 1.40 & 1.08 & 1.0 & 0.84 \\
& PID & 0.892 & 1.1 & 0.77 & 0.32 & - & 0.096 & 1.40 & 1.13 & 1.0 & 0.52 \\
\hline
\end{tabular}

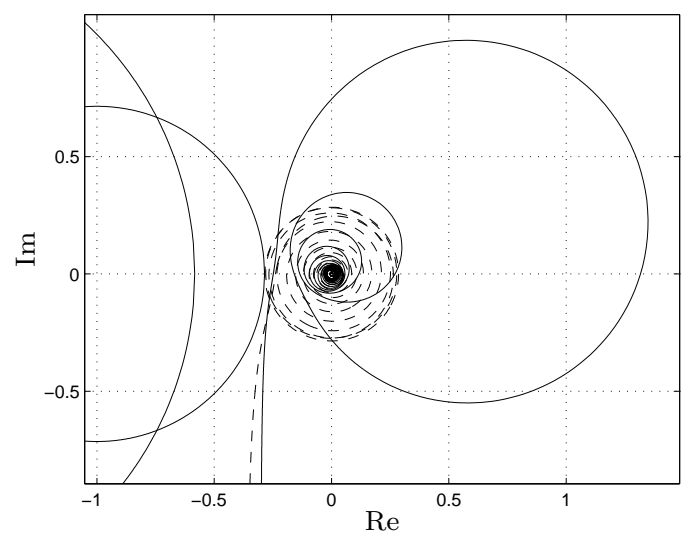

Fig. 5. Nyquist curves in Example I with PPI (-) and PID (--). Circles correspond to $\|S\|_{\infty}=\|T\|_{\infty}=1.4$.
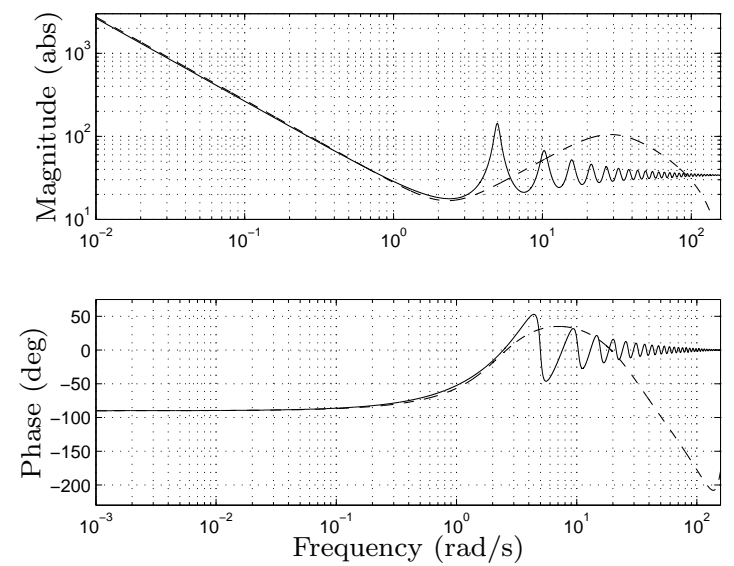

Fig. 6. Bode diagram of feedbacks in Example I with PPI (-) and PID (--).

crease over a wider frequency interval are better than over a narrow interval. This results in a 5\% lower IAE for the PID structure compared to the PPI structure.

\subsection{Example $I I: P_{9}, T=0.3$}

The optimal PPI controller with measurement filter for the process $P_{9}$ with $T=0.3$, have the robustness constraint $\left\|S_{\Delta}\right\|_{\infty} \leq M_{S}$ active prior the phase cross-over frequency when $\Delta L=\Delta \bar{L}$. Phase advance is given by the predictor, but is limited together with the gain as $\left\|S_{\Delta}\right\|_{\infty}=M_{S}$ at the negative real axis in the Nyquist diagram. The highest gain of the predictor is at the first peak. Since the process is of third order and the measurement filter time constant yields roll-off such that the noise sensitivity constraints are fulfilled, the Nyquist curve tends to 0 rapidly after the first predictor peak. The process may be approximated by a first order system with dead-time with static gain, time constant and dead-time approximately equal to 1,1 and 0.35 , respectively. From the controller parameters, it is seen that the design of the optimal PPI controller does not resemble model-matched design.
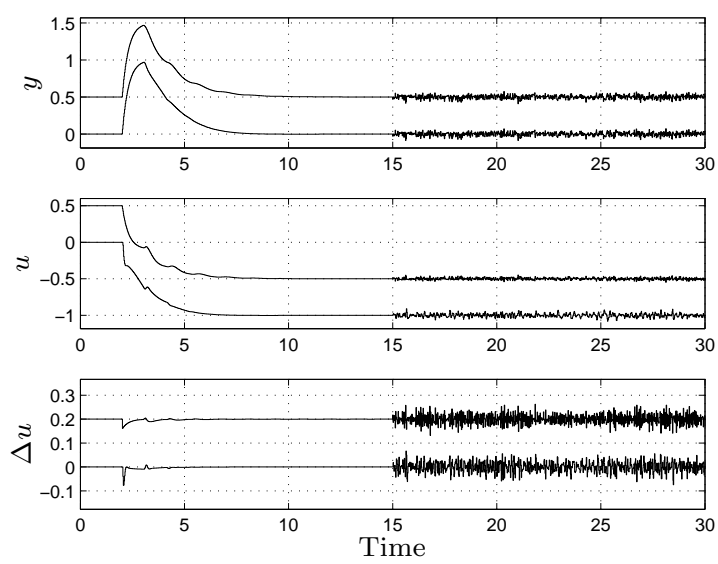

Fig. 7. Load disturbance responses in Example I with PPI (upper curves) and PID (lower curves). Top: Measurement signal. Middle: Control signal. Bottom: Inter-sample difference of control signal. Biases of 0.5 and 0.2 are added for separation.

The PID controller follows the phase advance of the PPI controller and has $\left\|S_{\Delta}\right\|_{\infty}=M_{S}$ for $\Delta L=\Delta \bar{L}$ prior the phase cross-over frequency. Compared to Example I, the PID controller may not increase its gain and phase over such a wide frequency interval above the phase crossover frequency due to the noise sensitivity constraints. The differences in increased gain and phase given by the two prediction methods are not as large as in Example I.

The optimal PPI structure yields a slightly higher gain cross-over frequency compared to the PID structure. It also has significantly higher gain at high frequencies. This yields the inter-sample sensitivity of the control signal due to noise to be larger, as seen in the load disturbance responses, but gives also a more rapid response to load disturbances. The PPI structure gives approximately $7.5 \%$ lower IAE than the PID structure. Hence, in this example, it is advantageous to use a feedback structure that may yield significant gain and phase advance peaks over a narrow frequency interval.

\section{SUMMARY}

This paper presented a comparison between PID and PPI controllers with associated measurement filters, considering load disturbance rejection, robustness towards process uncertainties and noise sensitivity of the control signal. For chosen optimization parameters and the majority of the processes in the batch, the performances of the two different structures, and hence the different prediction methods, are similar. However, for some processes with comparatively large time constants, the derivative part is allowed by the noise sensitivity constraints to yield phase advance and gain increase over a frequency interval wider than what is possible by the PPI predictor. This yields better performance by the PID than by the PPI. 


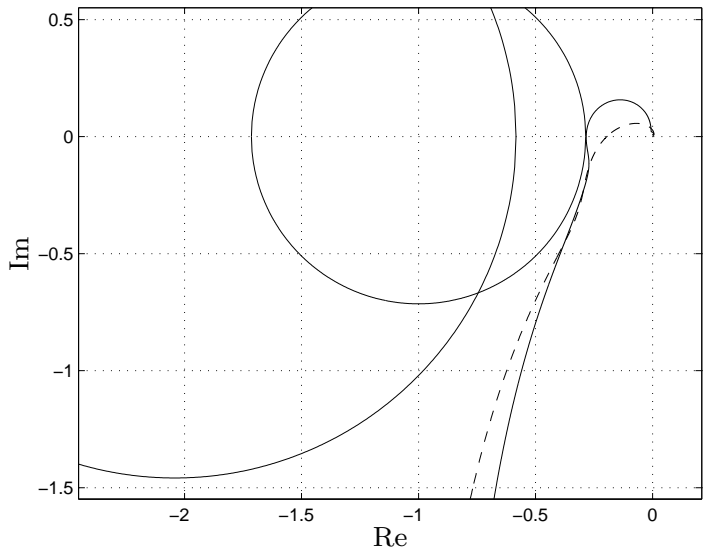

Fig. 8. Nyquist curves in Example II with PPI $(-)$ and PID (--). Circles correspond to $\|S\|_{\infty}=\|T\|_{\infty}=1.4$.
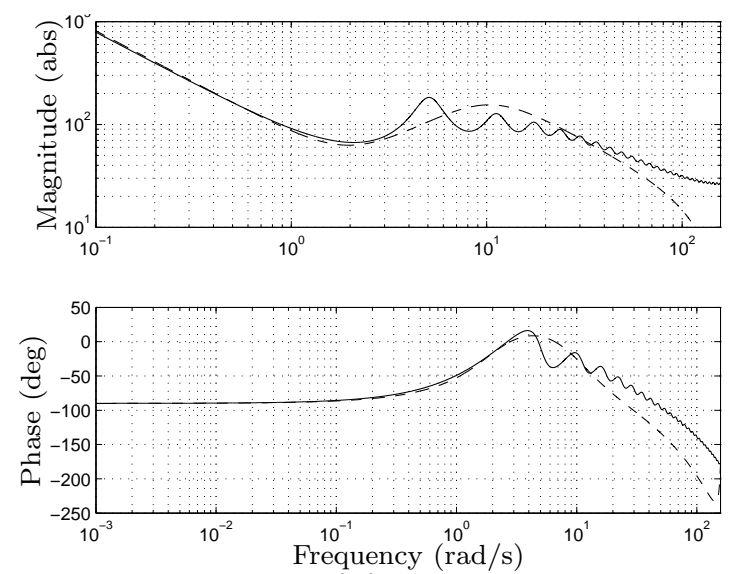

Fig. 9. Bode diagram of feedbacks in Example II with PPI (-) and PID (--).
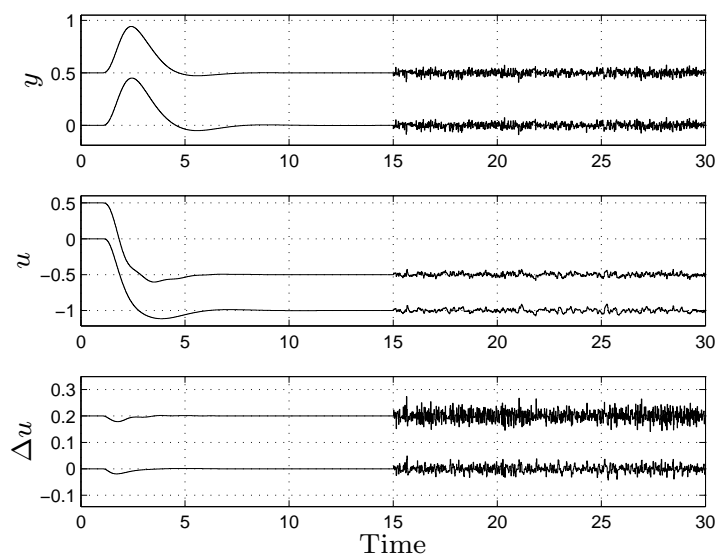

Fig. 10. Load disturbance responses in Example II with PPI (upper curves) and PID (lower curves). Top: Measurement signal. Middle: Control signal. Bottom: Inter-sample difference of control signal. Biases of 0.5 and 0.2 are added for separation.

\section{REFERENCES}

K.J. Åström and T. Hägglund. Advanced PID Control. ISA - The Instrumentation, Systems, and Automation Society, Research Triangle Park, NC 27709, 2006.

O. Garpinger. Design of Robust PID Controllers with Constrained Control Signal Activity. Licentiate thesis, Department of Automatic Control, Lund University, Sweden, March 2009.
T. Hägglund. An industrial dead-time compensating PI controller. Control Eng. Practice, 4:749-756, 1996.

A. Ingimundarson and T. Hägglund. Performance comparison between PID and dead-time compensating controllers. Journal of Process Control, 12:887-895, 2002.

A.J. Isaksson and S.F. Graebe. Derivative filter is an integral part of PID design. Control Theory and Applications, IEE Proceedings, 149(1):41-45, January 2002.

B. Kristiansson and B. Lennartson. Robust PI and PID controllers including Smith predictor structure. In Proceedings of the 2001 American Control Conference, volume 3, pages 2197-2202, 2001.

B. Kristiansson and B. Lennartson. Robust tuning of PI and PID controllers: Using derivative action despite sensor noise. IEEE Control Systems Magazine, 26(1): 55-69, 2006.

P. Larsson and T. Hägglund. Robustness margins separating process dynamics uncertainties. In Proc. of the European Control Conference 2009, August 2009.

P. Larsson and T. Hägglund. Control Signal Constraints and Filter Order Selection for PI and PID Controllers. In Proc. American Control Conference 2011, June 2011.

J.E. Normey-Rico, C. Bordons, and E.F. Camacho. Improving the robustness of dead-time compensating PI controllers. Control Eng. Practice, 5(6):801 - 810, 1997.

Z. Palmor. Stability properties of Smith dead-time compensator controllers. International Journal of Control, 32(6):937-949, 1980.

F.G. Shinskey. Feedback Controllers for the Process Industries. McGraw-Hill, 1994.

F.G. Shinskey. PID-deadtime control of distributed processes. Control Eng. Practice, 9(11):1177 - 1183, 2001.

O.J.M. Smith. Closed Control of Loops with Dead Time. Chemical Engineering Progress, 53:217-219, May 1957.

Appendix A. ROBUSTNESS CALCULATION

The robustness calculation method presented in Larsson and Hägglund [2009] is based on the open loop transfer function $C(s) F(s) G(s)$, where $C(s)$ is the controller, $F(s)$ is the measurement filter and $G(s)=G_{0}(s) e^{-s L}$ is the nominal process. The method considers a rotation of the Nyquist curve such that a point on the curve with amplitude close to 1 is rotated a certain angle. From the rotation, the corresponding change $\Delta L$ in dead-time can be calculated. When $G(s)$ is discretized using zero-order hold $(\mathrm{ZOH})$, then

$$
G_{0}(s) e^{-s L} \stackrel{\mathrm{ZOH}}{\longrightarrow} P_{0}(z) z^{-n}
$$

where $n=\lceil L / h\rceil, h$ is the sampling period, and $\lceil\cdot\rceil$ is the ceiling function. Adding $\Delta L$ to the time delay does not change $G_{0}(s)$, however, it may change $P_{0}(z)$ as

$$
G_{0}(s) e^{-s(L+\Delta L)} \stackrel{\mathrm{ZOH}}{\longrightarrow} \tilde{P}_{0}(z) z^{-m},
$$

where $m=\lceil(L+\Delta L) / h\rceil$. For the case $\Delta L=k h$, i.e., $\Delta L$ is an integer number of sample intervals, $\tilde{P}_{o}(z)=P_{o}(z)$ and $m=n+k$. However, for the case $\Delta L \neq k h, \tilde{P}_{o}(z)$ and $P_{o}(z)$ have minor differences in both phase and amplitude affecting the Nyquist curve. The differences are mainly at high frequencies close to the Nyquist frequency and if an appropriate sampling period is selected, the differences are negligible in the frequency interval wherein $\Delta L$ is computed. Hence, the method to calculate $\Delta L$ in Larsson and Hägglund [2009] may be used, yielding negligible errors. This is confirmed when analyzing the designed controllers and filters for the considered process batch. 